\title{
Stress effects of low-frequency stimulation on the c-Fos and endothelin- 1 changes in tree shrew
}

\author{
Farong Yu, Xiuzhen Lian, Mingren Xie*, Wen Yu, Lirong Guo, Zhennan \\ Zhang, Denglou Li, Shishuang Zhang \\ Key Laboratory of Evidence of Science and Technology Research and Application, Gansu \\ Province (Gansu Institute of Political Science and Law), Lanzhou,730070, China \\ *Corresponding author: Mingren Xie. Tel : 18693932139, Eail :tim9898@163.com
}

Keywords: Low-frequency stimulation, Tree shrew, Endothelin, Brain

\begin{abstract}
The chronic low-frequency stimulation could stimulate the expression of c-fos gene in the prefrontal cortex and enhance the endothelin-1 (ET-1) levels of liver and spleen in tree shrew (Tupaia). Our results suggested that application of chronic low-frequency stimulations yielded significant c-Fos protein expression in the prefrontal cortex of tree shrews. After $3 \mathrm{~h}$ of death, the average ET-1 concentrations of the live and spleen in both control and treatment groups were significantly decreased by $30.2 \%-20.5 \%$ and $21.3 \%-22.5 \%$, respectively. The ET- 1 levels of the liver and spleen are highly correlated over the elapsed time periods in both control and treatment groups. The significant enhancement of the c-fos gene expression and the ET-1 level suggested that c-fos gene and ET-1 play an important role in the defensive mechanisms against stress stimuli. The ET-1 postmortem changes could be used to estimate the postmortem intervals during the decay process.
\end{abstract}

\section{Introduction}

Stimuli that act as stressors could elicit a complex centrally coordinated response involving changes in mood, cognition, behavior, and endocrine output. This response has served as a useful experimental model of stress that has been applied to various animals to clarify molecular mechanisms responsible for stress responses. Responses to stressors involve activation of complex central nervous system pathways leading from stress perception to behavioral, autonomic, and endocrine responses.

Since the introduction of electrical shock treatment as a means in stress analysis, great interest has been manifested in the changes of brain and endocrine system. The previous studies showed that the coordination of the stress responses is governed by the nervous and endocrine systems, and that various low frequency stimulations and other environmental factors as stressors could induce pronounced physiological effects ${ }^{[1]}$, such as the induction the $\mathrm{c}$-fos gene expression and the release of endothelin ${ }^{[2]}$. The prefrontal cortex is one of the most important regions in the cerebral cortex that involves in the regulation and control of memory processes and emotional changes, and controls a person's ability to make decisions ${ }^{[3]}$. Analysis of the effects of a conditioned stressor on c-fos expression in prefrontal cortex has the potential of providing information about the neural substrates of stress.

ET-1 is a key mediator of vascular tone and renal homeostasis through antagonistic vasoactive effects and could be produced by many tissues, including liver and spleen. ET-1 exhibits a diverse set of biological activities and plays a significant role in stress responses. However, current understanding of the biochemical and molecular changes of c-fos gene and ET-1 associated with stress is equivocal and unresolved. No attempt has been made to investigate the changes of the concentrations of ET-1 in postmortem tissues, which could be used to estimate the postmortem interval or period since death in forensic data analysis.

Tree shrews are closely related to primates and human beings ${ }^{[4]}$ in morphology, physiology, and biochemical analyses. Because tree shrew has a high reproductive rate and is very sensitive to various stimulations and stresses of conditions, it is being considered as an appropriate experimental 
model in a variety of medical research fields, including metabolism, physiology, responses to stimuli, as well as effects of acute or chronic pressure on behaviors. This study was designed to investigate the effects of chronic low-frequency stimulation on the c-fos gene expression in the prefrontal cortical region and the ET-1 levels of liver and spleen, and to quantitatively determine the postmortem changes of ET-1 levels during decomposition of tree shrew cadavers. Our findings will provide valuable information for monitoring both the neuron-behavioral and cardiovascular responses, as well as estimating the postmortem interval in forensic science.

\section{Materials and Methods}

\section{Materials}

A total of 160 tree shrews (male) with an average weight of $120 \pm 5.0 \mathrm{~g}$ were obtained from the Experimental Animal Center of Kunming Institute of Zoology, The Chinese Academy of Sciences. Animals were housed 4 per cage in a temperature-controlled room at $24 \pm 1{ }^{\circ} \mathrm{C}$ and maintained on a 12:12 h light-dark cycle. They were fed with the standard pellet diet and water ad libitum during the entire experimental period. All experimental procedures involving animals were conducted according to the Institutional Guidelines for the Care and Use of Laboratory Animals. The kits used in this study included the RIA diagnostic kit (Beijing North Institute of Biotechnology), the avidin SP kit (Beijing Zhong Shan Biological Engineering Co., Ltd), and the rabbit-anti-rat c-fos detection kit (Wuhan Boster Biological Technology Co., Ltd.).

\section{Animal treatment}

160 tree shrews were randomly assigned to the control group and the stimulation group of 80 animals each. Foot-shock stress was applied in a foot-shock box with a grid floor connected to a shock generator and scrambler ${ }^{[5]}$. In the chronic stress experiment, each animal in the stimulation group was placed into a foot-shock stress box and electrically stimulated daily with electrical currents at a low frequency of $20 \mathrm{~Hz}$ and a pulse width of 0.30 microseconds at intensities of 0.15 $\mathrm{mA}$ for 10s duration footshock for 7 days. Control animals stayed undisturbed in their home cages throughout the experiment. One hour after initiation of the stressor on the $7^{\text {th }}$ day, animals in both control and stimulation groups were sacrificed by brief exposure to inhaled anesthesia (15s) with sodium pentobarbitone $(80 \mathrm{mg} / \mathrm{kg}$ i.p. $)$ as followed by decapitation. The tree shrew carcasses were left at room temperature in the animal house for further experiment.

\section{Sampling}

The tree shrew carcasses in each group were further randomly divided into 8 subgroups of 10 animals each. Samples of liver and spleen were collected at different time points $(1 \mathrm{~h}, 3 \mathrm{~h}, 6 \mathrm{~h}, 12 \mathrm{~h}$, $18 \mathrm{~h}, 24 \mathrm{~h}, 36 \mathrm{~h}$ and $72 \mathrm{~h}$ ) after death and processed for immunohistochemistry and quantitative analysis of protein expression. To ensure that no variation occurred across groups, sample tissues of animals from both groups were always processed at the same time.

\section{Detection of endothelin-1}

At each time point, $5 \mathrm{mg}$ of liver and spleen tissues from both groups were isolated and ground with $0.5 \mathrm{ml}$ of saline into homogenates, which were then centrifuged with a low-speed refrigerated centrifuge at 3,500 rpm for $10 \mathrm{~min}$. The resulting supernatants were collected and further processed using the RIA (radioimmunoassay) diagnostic kit. The levels of ET-1 were determined by a RIA $\gamma$ counter (SN-682B, the Shanghai Institute of Nuclear).

\section{c-fos gene expression assay}

At the $1 \mathrm{~h}$ time point, the brain was dissected and the tissue sfrom the prefrontal cortex were removed and fixed with $4 \%$ paraformaldehyde $-1.25 \%$ glutaraldehyde phosphate buffer $(\mathrm{pH} 7.4)$. The prefixed tissues were gradiently dehydrate-fixed with alcohol, embedded in paraffin, and sliced into $5 \mu \mathrm{m}$ thick. After rinsing, the sections were affixed onto chrome alum gelatinised slides and air dehydrated and coverslipped for immunohistochemical analysis. The prepared sections were 
dewaxed and then subjected to an antigen-retrieval protocol, which involved incubating the sections in microwave for $30 \mathrm{~min}$. Following this incubation the sections were treated with the avidin SP kit, followed by incubation with 1:200 final dilution of rabbit anti-rat c-Fos antibody for $24 \mathrm{~h}$ at $4^{\circ} \mathrm{C}$ in cfos immunohisto- chemistry. The resulting chromogen was detected using diaminobenzidinehydrogen peroxide (DAB color kit). Slides were then counterstained using hematoxylin, dehydrated through graded alcohols and xylene, photographed, and examined under a MRC 1024 confocal laser scanning microscope (BioRad).

\section{Image analysis}

Image analysis and densitometry were used to measure the overall changes in the labeled cells. Quantification of protein expression was determined by calculating the integrated optical density (IOD) and the mean absorbance (MA) of immunostained fields with each corresponding antibody. The optical density of a stain that recognizes a molecular constituent is directly proportional to the concentration of the molecule recognized by the stain. The IOD results are proportional to the mean optical density per area which reflects the concentration of immunoreactive antigen. While the MA, an indicator of cell viability, is used for evaluating the degree and depth of any staining. In this study, the c-fos immunostainings (10 slices from each group) were quantitied at X40 magnification using an Olympus BX-51 microscope with a Nikon Eclipse E-800 microscope. Image processing and data analyses were done using the YA-G5000 series of cell image analysis software.

\section{Statistical analysis}

The data were analyzed using the SPSS10.0 software. Statistically significant differences of the ET-1 levels between different conditions at each time point were determined by ANOVA. Significance was set at $p<0.05$.

\section{Results}

\section{Stress impact on the c-fos gene in the prefrontal cortex of tree shrew}

To elucidate the molecular events associated with stress in the prefrontal cortical region, we analyzed changes in the expression level of c-Fos protein. Our results suggested that application of chronic low-frequency stimulations yielded significant c-fos protein expression in the prefrontal cortex of tree shrews, characterized by substantial numbers of c-fos-positive, brightly labeled cells, compared with the levels of gene expression of control group (Fig.1). The data of the immunohistochemistry assays estimated by the total cell density based on the integrated optical density (IOD) and mean absorbance (MA) of immunostained fields (Table 1) showed that the IOD and MA values were significantly higher in the treated animals than those in the control animals.

\section{Effect of stress on the ET-1 levels in liver and spleen of Chinese tree shrew}

Results obtained in this study demonstrated that the treatment of low-frequency stimulation resulted in significant enhancement of the ET-1 contents of liver and spleen. The ET-1 levels in spleen were significantly higher than those in liver (Table 2). Compared to the tree shrews in control group, the ET-1 levels in the liver and spleen of the animals under the stress significantly increased by $25 \%$ and $23 \%$, respectively. After death, the changes of the ET-1 levels were gradually reduced at each time point. After $3 \mathrm{~h}$ of death, the average ET-1 concentrations of the live and spleen in both control and treatment groups were significantly decreased by $30.2 \%-20.5 \%$ and $21.3 \%-22.5 \%$, respectively. After $18 \mathrm{~h}$ of death, the average ET-1 levels of the live and spleen were decreased by more than $50 \%$ in both groups. The ET-1 levels of the liver and spleen are highly correlated over the elapsed time periods in both control and treatment groups (Fig. 2).

\section{Discussion}

Low-frequency stimulation is being considered as a stressor to study drug-resistant diseases, such as epilepsy neural activities ${ }^{[6]}$, and muscular activities in human and other animals. Long-term 
depression induced by low-frequent electric stimulation in the hippocampus, visual cortex, and motor cortex is though to be one of the main mechanisms underlying the inhibitory effects, and may be therapeutic in epilepsy. The present study revealed that the chronic treatment of low- frequency stimulation caused an elevated expression of c-fos gene in the prefrontal cortical region and an enhancement of the ET-1 levels of liver and spleen in tree shrews.

$\mathrm{C}$-fos gene belongs to the immediate early gene family of transcription factors, and immunolabelling for the expression of c-fos was used as a marker of neuronal activity elicited in brain by physiological, pharmacological, and environmental manipulations ${ }^{[7]}$. C-Fos expression is very low in most neurons under basal conditions, but can be rapidly and transiently enhanced in response to external stimuli. Our results showed that application of prolonged low frequency stimulation significantly affected the c-Fos expression in prefrontal cortical region of tree shrews, characterized by substantial numbers of c-Fos positive, brightly labeled cells, compared with the levels of c-Fos expression in the control group (Fig. 1). The similar results were also reported in the isolated rat mesenteric arteries, the singulate, piriform, and other regionsof rats, when animals or sample tissues were exposed to the environmental stressors.

The prefrontal cortex function is related to the regulation and control of memory processes and emotional changes, and involved in executive functions and the expression of personality. It has been clearly established that the prefrontal cortex serves both cognitive and emotional functions ${ }^{[8]}$. The increased c-Fos protein observed in the present study (Fig. 1b and Table 1) postulated that continued stressful circumstances, including the low-frequency electric stimulation with optimal intensity and various other stress-inducing factors can potentiate the c-Fos expression in the prefrontal cortex, suggesting that c-fos gene plays a role in the defensive mechanisms against stress stimuli and the prefrontal cortical regions of tree shrew may undergo plastic changes following a chronic treatment of stimulation.

Endothelins include three isoforms (known as ET-1, -2, -3) with varying regions of expression and exert a variety of biological, physiological, and metabolic actions through two types of receptors $\left(\mathrm{ET}_{\mathrm{A}}\right.$ and $\left.\mathrm{ET}_{\mathrm{B}}\right)$. They are normally kept in balance in the circulation that are likely not sufficient to activate cellular processes, but are over-expressed in response to hypoxia, pro-inflammatory cytokines, bacterial toxins, and other external stresses. ET-1 is a potent endothelium derived vasoactive peptide that plays a central role in regulating vascular tone and influences various organ functions in mammals ${ }^{[9]}$. Acute stimulation of the environmental stressors can cause the significant changes of the blood and bone marrow in tree shrew. Our results confirmed that chronic lowfrequency stimulation significantly enhanced the ET-1 levels in both liver and spleen by $25 \%$ and $23 \%$, respectively (Table 2), which are consistent with the results reported in previous studies. ET-1 production is stimulated by many factors, including endotoxin, epinephrine, transforming growth factor b1, angiotensin II, and arginine-vasopressin.

The level of circulating ET-1 has been shown to increase significantly in conditions such as renal failure, congestive heart failure, and endotoxic shock. Many processes affecting the liver also involve the spleen due to reticuloendothelial system involvement. Spleen is one of the major sites of ET-1 release in cirrhotic patients. Although a significant hepatic expression of ET-1 was observed during periods of endotoxin exposure in rat, our study demonstrated that spleen relased more ET-1 production than liver (Table 2). The up-regulated ET-1 levels in liver and spleen suggested that ET1 plays an important role in the prevention of stress stimuli.

Another interesting finding in this study was the determination of the postmortem changes of the ET-1 in the liver and spleen of tree shrew. Analyzing forensic data, as a general rule, is a timecritical process. Forensic scientists have long used the bodies of pig, rabbit mouse, and the population of insects as potential surrogates to human models to simulate the changes occurring in a cadavar over time, in order to work out how long a corpse has been lying undiscovered. Decomposition is a continual process of gradual decay and disorganization of organic tissues and structures after death. The combination of chemical and optical methods proved very useful and informative, uncovering hidden aspects of the early stages of decay and also guiding in the development of combined chemical and imaging methods for the detection of dead bodies. 
The common forensic approaches for evaluating the time of death, such as looking at rigor mortis, loss of body heat, and pooling of blood inside the body, are useful only for the first two or three days after death and require more complex analytical methods. Our study suggested that the postmortem changes of the ET-1 concentrations in the liver and spleen tissues can be used to determine the postmortem intervals or times since death and beneficial to numerous applications of forensic science. From our results, after death, the ET-1 levels of the liver and spleen in both control and treatment groups were gradually reduced at each time point (Fig. 2, Table 2). The average ET-1 levels in live and spleen were decreased by $50 \%$ after $18 \mathrm{~h}$ of death. The previous forensic studies also suggested that a 2-h infusion of lipopolysaccharide (LPS) endotoxin could cause a significant elevation of ET-1 in the hepatic portal vein, which could persist for several hours after termination of the infusion.

Table 1. Effect of low-frequency stimulation on the expression of c-FOS protein in the cerebral cortex of Chinese tree shrew(Mean \pm S.D, $n=10$ )

\begin{tabular}{ccc}
\hline Index & Treatment group & Control group \\
\hline IOD & $11.06 \pm 1.3^{\mathrm{A}}$ & $6.21 \pm 1.4$ \\
MA & $0.24 \pm 0.02^{\mathrm{a}}$ & $0.16 \pm 0.03$ \\
\hline
\end{tabular}

${ }^{\mathrm{a}} P<0.05$ vs Control group; ${ }^{\mathrm{A}} P<0.01$ vs Control group

Table 2. Postmortem changes of endothelin-1 levels of liver and spleen at different time points in Chinese tree shrew $\left(10^{2} \mathrm{pg} / \mathrm{ml}, \mathrm{n}=10\right)$

\begin{tabular}{ccccccccc}
\hline \multicolumn{4}{c}{ Control group } & \multicolumn{5}{c}{ Treatment group } \\
\hline $\begin{array}{c}\text { Time } \\
\text { point }\end{array}$ & Liver & $\begin{array}{c}\text { Change } \\
(\%)^{\#}\end{array}$ & Spleen & $\begin{array}{c}\text { Change } \\
(\%)^{\#}\end{array}$ & Liver & $\begin{array}{c}\text { Change } \\
(\%)^{\#}\end{array}$ & Spleen & $\begin{array}{c}\text { Change } \\
(\%)^{\#}\end{array}$ \\
\hline $1 \mathrm{~h}$ & $166.23 \pm 3.4$ & & $797.16 \pm 2.3$ & & $206.63 \pm 2.4^{\mathrm{A}}$ & & $976.32 \pm 3.2^{\mathrm{A}}$ & \\
$3 \mathrm{~h}$ & $116.06 \pm 2.2$ & 30.2 & $634.13 \pm 2.6$ & 20.5 & $162.63 \pm 4.6^{\mathrm{A}}$ & 21.3 & $756.22 \pm 3.2^{\mathrm{A}}$ & 22.5 \\
$6 \mathrm{~h}$ & $109.31 \pm 6.2$ & 34.2 & $562.13 \pm 2.2$ & 29.5 & $141.11 \pm 2.6^{\mathrm{A}}$ & 31.7 & $672.31 \pm 2.2^{\mathrm{A}}$ & 31.1 \\
$12 \mathrm{~h}$ & $86.14 \pm 0.4$ & 48.2 & $457.22 \pm 2.4$ & 42.6 & $106.27 \pm 1.4^{\mathrm{A}}$ & 48.6 & $554.42 \pm 2.4^{\mathrm{A}}$ & 43.2 \\
$18 \mathrm{~h}$ & $53.47 \pm 0.2$ & 67.8 & $393.22 \pm 2.4$ & 50.7 & $85.32 \pm 1.2^{\mathrm{A}}$ & 58.7 & $476.23 \pm 3.3^{\mathrm{A}}$ & 51.2 \\
$24 \mathrm{~h}$ & $39.33 \pm 0.3$ & 76.3 & $238.25 \pm 2.4$ & 70.1 & $49.64 \pm 1.3^{\mathrm{A}}$ & 76.0 & $307.16 \pm 2.4^{\mathrm{A}}$ & 68.5 \\
$36 \mathrm{~h}$ & $31.65 \pm 0.4$ & 81.0 & $196.16 \pm 1.4$ & 75.4 & $41.23 \pm 1.3^{\mathrm{A}}$ & 80.0 & $264.32 \pm 2.2^{\mathrm{A}}$ & 72.9 \\
$72 \mathrm{~h}$ & $27.62 \pm 0.2$ & 83.4 & $163.13 \pm 2.2$ & 79.5 & $36.94 \pm 1.2^{\mathrm{A}}$ & 82.1 & $241.24 \pm 2.2^{\mathrm{A}}$ & 75.3 \\
\hline
\end{tabular}

${ }^{\mathrm{A}} P<0.01$ vs Control group;;: The decrease of the ET-1 level at each time point relative to the $1 \mathrm{~h}$ level.

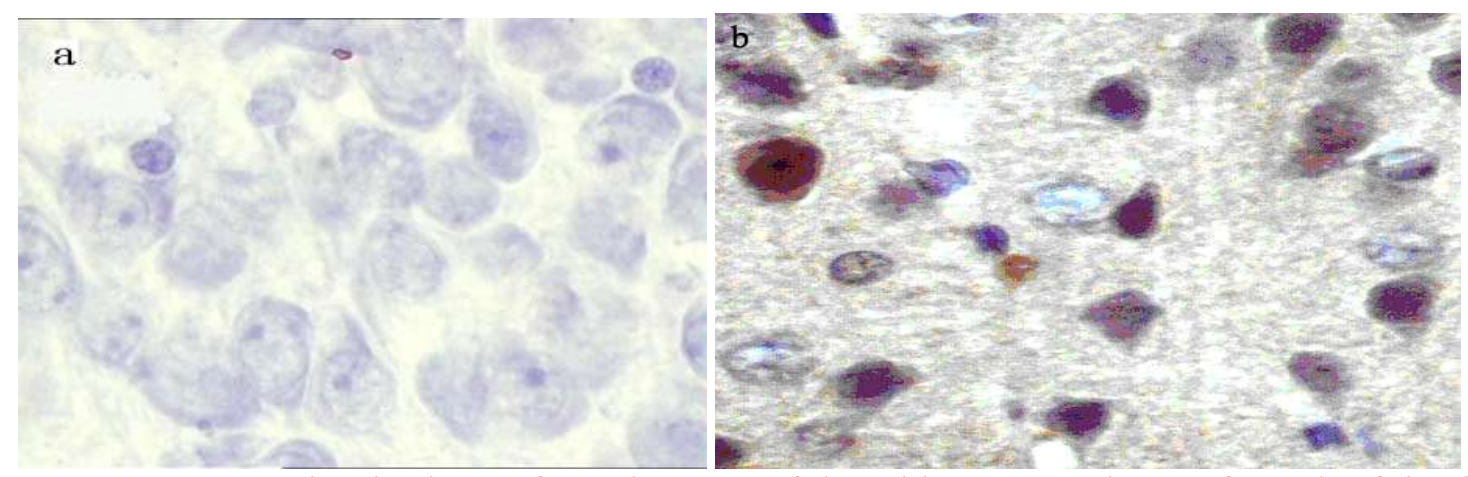

Fig. 1. C-Fos expression in the prefrontal cortex of the Chinese tree shrew after $1 \mathrm{~h}$ of death. a: control group, $\mathrm{x} 400$; b: treatment group, $\mathrm{x} 400$ 

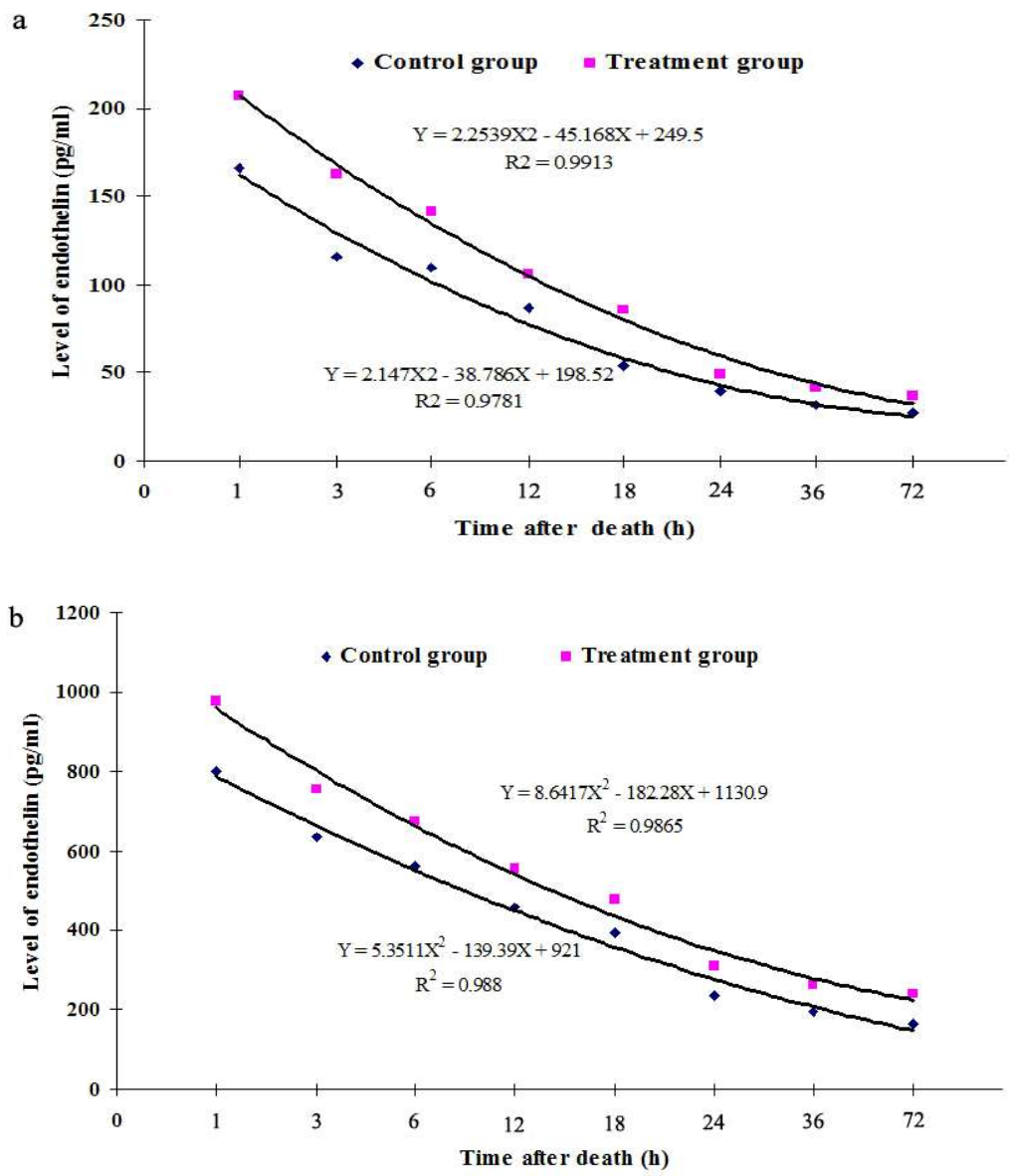

Fig. 2. Postmortem changes of the endothelin-1 (ET-1) levels (Y-axis) of liver and spleen at different time points (X-axis) in Chinese tree shrew. a: liver, b: spleen

\section{Conclusion}

This study provides the first documentation of the postmortem changes of the ET-1 concentrations in liver and spleen tissues of tree shrew. As a stressor, the low-frequency stimulation could stimulate the expression of c-fos gene of the prefrontal cortical region and the enhancement of the ET-1 levels of liver and spleen. The postmortem ET-1 redistribution could be used for determing the postmortem intervals and estimating the differences of the ET-1 concentrations of the normal and treated deceased subjects.

\section{Acknowledgements}

The research work was supported by National Natural Science Foundation Project Grant No.:31060283; National Social Science Foundation of China No.: 07BSH 054; Gansu Province Groups of Basic Research Innovation Projects No.: 145RJIA333;Gansu Province Science and Technology Support Projects No.: 1304FKCA082; Key Laboratory of Evidence of Science and Technology Research and Application, Gansu Province, Plan Projects No.: GZF2014XZDLW18.

\section{References}

[1] K.Jung, D.Lelic, S. Rottmann, A.M. Drewes, Electrical low-frequency stimulation induces central neuroplastic changes of pain processing in man. Eur. J. Pain, 16(2012) 509-521.

[2] F.R. Yu, X.Z. Lian, S.T. Feng, Effects of the external environment pressure on the Chinese tree shrew reproductive physiology. Chin. J. Pathophysiol. 23(2007) 1808-1810. 
[3] E.K. Miller, J.D. Cohen, An integrative theory of prefrontal cortex function. Ann. Rev. Neurosci. 24 (2001) 167-202.

[4] E. Fuchs, G. Flugge, Social stress in tree shrews: effects on physiology, brain function, and behavior of subordinate individuals. Pharmacol. Biochem. Behavior 73(2002) 247-258.

[5] A. Trentani, S.D. Kuipers, J.A. den Boer, Immunohistochemical changes induced by repeated footshock stress: revelations of gender-based differences. Neurobio. Disease, 14(2003) 602-618.

[6] O. Klavir, R. Genud-Gabai, R. Pax, Low-frequency stimulation depresses the primate anteriorcingulate-cortex and prevents spontaneous recovery of aversive memories. J. Neurosci. 32(2012) 8589-8597.

[7] M. VanElzakker, R.D. Fevurly, T. Breindel, R.L. Spencer, Environmental novelty is associated with a selective increase in Fos expression in the output elements of the hippocampal formation and the perirhinal cortex., Learning Memory 15(2008) 899-908.

[8] J.D. Steele, S.M. Lawrie, Segregation of cognitive and emotional funciton in the prefrontal cortex: a stereotactic meta-analysis.Neuroimage 21(2004) 868-875.

[9] P.W. Angus, Role of endothelin in systemic and portal resistacne in cirrhosis. Gut, 55(2006) 1230-1232. 